\title{
PSYCHOSOCIAL PREDICTORS FOR THE INTENTION OF BRAZILIAN ELDERLY IN RECEIVING THE VACCINE AGAINST COVID-19
}

Thales Coutinho ( $\square$ thales.coutinho@hotmail.com )

Faculdade Estácio https://orcid.org/0000-0002-7968-0154

Fernando Assunção

Clínica +60 saúde

Mariana Dias

Clínica + 60 Saúde

Thiago Moraes

Centro Universitário Campos de Andrade

Natália Dias

Universidade Federal de Santa Catarina

Article

Keywords:

Posted Date: March 25th, 2021

DOl: https://doi.org/10.21203/rs.3.rs-362316/v1

License: (9) This work is licensed under a Creative Commons Attribution 4.0 International License. Read Full License 


\section{Abstract}

Once the immunizers are already available in the COVID-19 Pandemic, there is a need to identify the psychosocial factors that increase the positive attitude towards vaccines, particularly among the elderly. We investigated the role of psychosocial variables, which could affect the attitude towards the vaccines. Factors such as: uncertainty in relation to the vaccine, fear of COVID-19, political orientation, precisely the intention among the Brazilian elderly $(>60$ years, $n=328$ ) to be vaccinated. There was a more positive intention towards Oxford-AstraZeneca in relation to Sinovac-CoronaVac. For the Oxford-AstraZeneca vaccine, less uncertainty regarding vaccines and greater fear of COVID-19 led the study to predict $26.6 \%$ of the intention to use it. In the case of Sinovac-CoronaVac, in addition to the previous variables, a leftwing political orientation led to a prediction of $44.3 \%$ in the intention to use it. It was also highlighted the importance of reducing uncertainty about vaccines in public campaigns to achieve older adults' mass vaccination.

\section{Introduction}

One of the best predictors of the impact of COVID-19 at national levels is the percentage of the local elderly population (1), once older patients affected by COVID-19 have a less favorable prognosis (2). Among other reasons, this is due to the higher incidence of comorbidities, such as hypertension and diabetes (3) in this portion of the population.

The Brazilian population pyramid has aged over the past few decades (4). Therefore, it is necessary to study the attitudes of the elderly towards vaccines to support evidence-based public campaigns. This is important because, even the elderly showing greater vulnerability to the COVID-19, not necessarily assume adaptive behaviors during the pandemic (5), making vaccination even more important to prevent mortality.

However, the vaccination coverage for adults and the elderly decreased dramatically over the past few years $(6 ; 7)$. In a recent survey conducted with elderly Brazilians, the researchers identified that the main reasons for older adults with arterial hypertension to hesitate to take the influenza vaccine were: the fact that they rarely have a flu, distrust in the vaccine efficacy, and fear of side effects (8). In the specific case of the vaccine against COVID-19, one of the leading causes of the hesitation in taking them is the belief that the vaccine is unsafe (9). Therefore, it is crucial to assess the impact of individual differences in beliefs about vaccines, such as distrust concerning the "record time" in which the scientists developed vaccines against COVID-19 and their future side effects, because this may impact the intention to vaccinate.

Thus, fear can be a relevant emotion, especially in contexts of real threat (10), for having the property of triggering a more cautious response (11) and reducing risk behaviors (12). In the case of a pandemic, the most significant risk is contamination, especially in people who are more vulnerable to complications 
from the disease. Therefore, at first, the fear of becoming ill could favor a greater intention to get vaccinated as a cautious strategy to reduce death risk.

The "fear of COVID-19" was initially conceptualized, and measured by Ahaosu and collaborators (13). This specific type of fear demonstrated a high correlation with levels of depression, anxiety, and the perceived vulnerability to the disease. A study conducted by Shook and collaborators (14) demonstrated that the perceived vulnerability to the disease is related to more preventive behaviors against the virus.

As the vaccine is also a preventive measure (15), we hypothesize that the fear of COVID-19 may favor a more favorable attitude towards the use of vaccines. After all, in another study, the fear of COVID-19 was the only predictor of more positive behaviors during the pandemic, such as the habit of washing hands with soap and water and maintaining social distance (16).

On January 17th, 2021, Brazil applied its first dose of vaccine against COVID-19 in São Paulo. Since then, other states have mobilized to start vaccinating the population, giving preference to frontline health professionals and the elderly, according to international guidelines (17). Vaccination in the Brazilian territory runs slowly due to several problems, including logistical ones (18) and scientific denialism (19). For this reason, we believe it is also relevant to investigate the impact that political orientation may have on the intention to vaccinate against COVID-19, based on previous studies that demonstrated that the pandemic is politicized since its beginning (20). Also, there is evidence that vaccine hesitancy is greater among those who define themselves as having a right-wing political orientation (21).

Thus, it is relevant to identify the role of psychosocial factors, including the fear of COVID-19 and uncertainty regarding the vaccine and political orientation, in the elderly population's intention to be vaccinated (22). The hypothesis is that the combination of a greater fear of COVID-19, less uncertainty concerning the vaccine, and a left-wing political orientation may increase the two vaccines' endorsement: Oxford-AstraZeneca (modified adenovirus) and Sinovac-CoronaVac (inactivated live virus).

\section{Method}

\section{Sample:}

A total of 589 individuals participated in the research. However, 235 of them were 59 years old or less and were excluded. Also, 26 of the remaining participants reported having a neurological/psychiatric diagnosis and were excluded. Therefore, there were 328,252 of whom $76.8 \%$ were female $(n=328,76.8 \%$ female). The respondents' average age was 69.67 years (SD: 7.841), ranging from 60 to 92 . Regarding the level of education, $47(14.3 \%)$ had completed elementary school, $72(21.9 \%)$ had completed high school, 26 (7.9\%) had incomplete higher education, 96 (29.2\%) had completed higher education and 87 $(26.5 \%)$ had incomplete or complete graduate degrees. Regarding the socioeconomic class, they were divided according to the monthly income range, following the rules of the Brazilian Institute of Geography and Statistics. Namely, 83 (25.3\%) belong to class E, 94 (28.6\%) to class D, 99 (30.1\%) to class C, 41 
(12.5\%) to class B and 11 (3.3\%) to class A. Among the participants, 27 (8.2\%) had COVID-19 and 246 (75\%) had a family member or close friend affected by the disease.

\section{Measures:}

Fear of COVID-19 Scale: A self-report instrument consisting of seven items. The respondent must judge each item, using a Likert scale, ranges from 1 (strongly disagree) to 5 (strongly agree). The scale was developed initially by Ahorsu and collaborators (23), and independent Brazilian studies corroborated its psychometric quality, endorsing a unifactorial structure $(24 ; 25)$. A higher total score is associated with greater fear of COVID-19.

Political self-identification: to investigate the political orientation of the participants, we adopted a method analogous to that used by Inbar and collaborators (26). After the question "What do you consider your orientation to be, on the political spectrum?" the individual should check any of the following options on a gradual scale of the political spectrum: 1) extreme left; 2) left; 3) middle-left; 4) middle; 5) middleright; 6) right; and 7) extreme right.

Vaccine Uncertainty Index: the participant answered two questions, using a Likert agreement scale that ranged from 1 (not at all) to 5 (totally). One of the questions was about the uncertainties regarding the vaccine development process: "How much does the fact that the vaccine against COVID-19 is being developed in record time, without precedents, negatively affect your view on it?". The second question was about the possible side effects: "How much does the fact that we don't know the long-term side effects of the future vaccine against COVID-19 negatively affect your view of it?". For this index, we consider the average of the answers to these two questions. Higher scores indicate more uncertainty concerning the vaccine.

Vaccination intention questionnaire: In Brazil, only two vaccines are available to the population at the moment of the survey: Oxford-AstraZeneca and Sinovac-CoronaVac. The participant was presented with two questions, asking him to inform how much he was willing to receive the Oxford-AstraZeneca vaccine and the Sinovac-CoronaVac vaccine. Both questions adopted the same gradation on the Likert scale, ranging from 1 ("I would not take it under any circumstances") to 7 ("I would take it for sure").

Sociodemographic questionnaire: a brief questionnaire to identify the participants' sociodemographic characteristics, such as sex, age, education level, income, if the participant had Covid-19, and if a family member or close friend had Covid-19.

\section{Proceedings:}

This research was submitted and approved by the Institutional Review Board of the Federal University of Santa Catarina, Brazil (CAAE: 38794920.0.0000.0121).

We conduct the research entirely online, using the Google Form platform. The link to the GoogleForms was sent through social media. The survey started on January 20th, 2021 and ended on February 15th, 
2021.

Participants completed the forms voluntarily, without receiving any financial compensation. Still, we don't ask for data that could generate personal identification, such as name or e-mail. We adopt this strategy to avoid the social desirability bias.

After acceptance of the Informed Consent Term, the instruments were presented. After submitting the form, the participant received a message of acknowledgment, and their participation was ended.

\section{Analysis:}

A Student's paired t-test was performed to compare the levels of intention to be vaccinated with each vaccine, Oxford-AstraZeneca and Sinovac-CoronaVac. It allowed us to define whether the subsequent analyzes would use a single index of intention to vaccinate or whether it would be pertinent to conduct the analyzes separately. An analysis of variance was conducted to verify the effect of education level on the level of intention to be vaccinated with each vaccine. For both analyzes, as there was no normal distribution of data, bootstrapping procedures were performed (1000 re-samples; $95 \% \mathrm{Cl} \mathrm{BCa).}$

Based on the results, we conduct two multiple linear regression analyzes (forward method) to test explanatory models of the intention to be vaccinated with 1) Oxford-AstraZeneca and 2) SinovacCoronaVac. For both analyzes, we consider the following variables as predictors: sex, age, if the participant had COVID-19, if a family member or close friend had Covid-19, vaccine uncertainty index, political orientation, and the score on the Fear of COVID-19 Scale.

For both models generated, the assumptions of independence between IV's (multicollinearity: Tolerance and VIF) and independence between residues in the sample (Durbin-Watson) were met, with adequate values. We do not identify outliers (via standardized residues, Cook's Distance and Mahalanobis Distance) that could harm the models. For these analyzes, we use SPSS 20. We also carried out moderation analyses to investigate the extent to which the political orientation would moderate the relationship between Fear of COVID-19 and the Vaccine Uncertainty Index with intention to vaccinate, especially in the case of the Sinovac-CoronaVac. In case of significant interaction, non-standardized coefficients $(b)$ were reported. Process macro (v3.5) for SPSS was used.

\section{Results}

Initially, it was verified, via paired t-test, that there was a statistically significant difference $(t[327]=8.256$; $p<0.001)$ in the intention to vaccinate when considering the Sinovac-CoronaVac vaccines $(M=5.30 ; S D=$ $2.28)$ and Oxford-AstraZeneca $(M=6.05 ; S D=1.76)$. The effect size of the difference was small to medium (Cohen's $d=0.46$ ). Because of this, it was decided to conduct the subsequent regression analyzes considering the vaccines separately. There was no effect of education level on the intention to vaccinate either with Sinovac-CoronaVac $(F[4,327]=1.013 ; p=0.401)$ or with the Oxford-AstraZeneca vaccine $(F[4,327]=0.314 ; p=0.869)$. 
Table 1 shows the model obtained considering the Vaccination intention questionnaire for the OxfordAstraZeneca vaccine. The results demonstrated that there is a significant influence of the variable Vaccine Uncertainty Index $(F[1,326]=112.834, p<0.001)$ and, together with this, the Fear of COVID-19 in the intention to be vaccinated $(F[2,325]=60.183, p<0.001)$. The first model considered only the variable Vaccine Uncertainty Index and explained $25.5 \%$ of the variance of the intention to be vaccinated (OxfordAstraZeneca). The second model included the variable Fear of COVID-19 and raised the explanatory power to $26.6 \%$. Despite the small difference in terms of explanatory power, $\Delta R^{2}=1.3 \%$ was significant $(p=0.016)$. Table 1 shows the coefficients for significant predictors. The sociodemographic variables, such as sex, age, and being diagnosed with COVID-19, having family or friends with COVID-19, and political orientation did not significantly impact the prediction model.

Table 2 shows the model obtained considering the Vaccination intention questionnaire for the SinovacCoronaVac vaccine. The results demonstrated a significant influence of the variable Vaccine Uncertainty Index $(F[1,326]=249.023, p<0.001)$, Vaccine Uncertainty Index and Political orientation $(F[2,325]=$ $130.999, p<0.001)$, and these together with Fear of COVID-19 $(F[3,324]=89.712, p<0.001)$ in the intention to be vaccinated, all models with a significant adjustment. The first model considered only the variable Vaccine Uncertainty Index and explained $43.3 \%$ of the variance of the intention to be vaccinated (Sinovac-CoronaVac). The second model included the variable political orientation and raised the explanatory power to $44.3 \%$, with $\Delta \mathrm{R}^{2}=1.3 \%$ and significant $(p=0.006)$. The latter model also integrated the Fear of COVID-19 variable, with an explanatory power of $44.9 \%$. In relation to the previous one, the $\Delta \mathrm{R}^{2}=0.7 \%$ was again significant $(p=0.037)$. Table 2 shows the coefficients for the predictors that entered the model. The sociodemographic variables, such as sex, age, and having been diagnosed with COVID-19, and having family or friends with COVID-19 did not significantly impact the prediction model.

Given the specificity of the effect of political orientation in the vaccination intention with SinovacCoronaVac, we decided to better understand this interaction by testing moderation models. The interaction between Fear of Covid-19 and political orientation did not have a statistically significant effect ( $\mathrm{B}=0.017, t=1.38, p=0.16)$, indicating no moderation effect. However, the interaction between Vaccine Uncertainty Index and political orientation had a statistically significant effect $(\mathrm{B}=0.114, t=2.379, p=$ 0.017), indicating a moderation effect.

For all levels of political orientation (trend on the left - cutoff point at $16^{\text {th }}$ percentile or the $16 \%$ lower in distribution; more central trend - cutoff point at median - $64^{\text {th }}$ percentile; trend on the right - cutoff point from $84^{\text {th }}$ percentile $-16 \%$ higher), there was a significant and negative relationship between Vaccine Uncertainty Index and vaccination intention, but with different magnitudes (respectively, for each politically oriented cut-off point: $b=-0.61, p<0.001 ; b=-0.84, p<0.001 ; b=-1.67, p<0.001)$. Figure 1 illustrate the effects obtained.

\section{Discussion}


The previous literature has already demonstrated that it is necessary to evaluate the efficacy and possible side effects of each of the many vaccines against COVID-19 separately (27). In our case, given the difference observed between the levels of intention to be vaccinated with each vaccine, prediction models were tested for each one separately. There was no effect of the level of education on the intention to be vaccinated. Still, the results showed that there is a greater intention to be vaccinated using the OxfordAstraZeneca vaccine. This result sets a precedent for future research, interested in evaluating the will to be immunized against COVID-19 and considering separately the vaccines offered in each country.

In addition to the more favorable intention to the Oxford-AstraZeneca vaccine, the analyses showed that each vaccine's intention also has some different predictive factors. For example, smaller Vaccine Uncertainty Index and greater Fear of COVID-19, in this order, were associated with a greater intention to get this vaccine. These variables explained up to $26.6 \%$ of the will to take the Oxford-AstraZeneca vaccine. In contrast, sociodemographic variables, such as sex, age, having a previous diagnosis of COVID-19, or a family member or close friend with COVID-19, and political orientation, had no significant effect on the model.

Concerning the Sinovac-CoronaVac vaccine, Vaccine Uncertainty Index, political orientation, and Fear of Covid-19, in this order, explained up to $44.3 \%$ of the intention to use it. More specifically, smaller uncertainty, a left-wing politics identification, and greater fear of COVID-19 predict greater intention to undergo vaccination. At the same time, the other variables did not have any impact.

Only the Vaccine Uncertainty Index explains a robust portion of the model variance (approximately $25 \%$ for the intention to be vaccinated with Oxford-AstraZeneca and $43 \%$ with Sinovac-CoronaVac), even though the role of Fear of COVID-19 is also significant in both models. The belief that SARS-CoV-2 vaccines are unsafe has already been considered one of the main causes of vaccine hesitancy (28). Also in the case of influenza, aspects such as disbelief in vaccine efficacy and fear of side effects are factors associated with older adults' hesitation to get vaccinated (29). In addition to these findings, our result suggests that insecurity about unknown side effects, and about the "record time" of this vaccine development, may be risk factors for achieving the mass immunization.

The Fear of Covid-19 also significantly affected both models, although its contribution was relatively small. Others also support this finding regarding the role of perceived vulnerability to prophylactic behavior $(30 ; 31)$. In sum, fear appears to have an important adaptive role (32-34) that, in this pandemic context, can contribute to the vaccine intention.

One of the differences between the models is that although Vaccine Uncertainty Index's role is relevant in both, its contribution is more significant in predicting the intention to vaccinate with Sinovac-CoronaVac. This could be explained given the greater variability in responses to the intention directed to this vaccine, which impacted the associations' strength. Still, Sinovac-CoronaVac was the only vaccine whose intention to use had some impact from the political orientation. This effect, although small, should not be disregarded. Unlike other world political leaders, Jair Bolsonaro (president of Brazil) tends to endorse measures that go against international health agencies' recommendations (35). Sinovac-CoronaVac, for 
example, was, at a certain time, target of his criticism. Therefore, it makes sense that those respondents whose political orientation is closer to that of the president (extreme right) also have a less favorable attitude towards the use of Sinovac-CoronaVac.

Still concerning Sinovac-CoronaVac vaccine and the effects of political orientation, the relationship between the variables shows that less Vaccine Uncertainty Index is associated with a greater vaccination intention. However, this relationship is more robust in the more right-wing political orientation spectrum. Interestingly, when uncertainty is low, there is a high intention to be vaccinated, regardless of political orientation. In contrast, when uncertainty is high, differences in intention as a function of political orientation become more evident. This may suggest that information (reduction of uncertainty) may be an effective strategy to overcome political orientation's effect.

These results present contributions to the vaccination campaigns against COVID-19, mainly with a focus on elderly populations. First, campaigns should focus on making vaccine safety clear to reduce uncertainty about them, especially concerning their development process and the scientific rigor of that process. For example, in the case of vaccines available in Brazil, both have already been shown to have a high level of safety $(36 ; 37)$, although Sinovac-CoronaVac has not yet demonstrated robust data related to the elderly population (38).

In this context, health professionals and science communicators, who will have a very relevant role in this campaign, must be very transparent in their communications, avoiding dubious information (39). Also, another important factor is the effect that the news's focus can trigger on the spectator (40). For example, although the record time with which it was possible to develop the vaccine is a scientific milestone, this factor seems to arouse a negative attitude in the general population. Therefore, instead of focusing on the "speed of development of vaccines", the media should clarify how all the steps have been taken to ensure a safe and efficient vaccine. In other words, it is necessary to make clear the nature of the scientific method itself to reduce misinformation and the cognitive biases that predispose to denialism and vaccine hesitancy (41).

The positive impact of fear, although smaller, is also relevant and should be considered in the planning of public campaigns aimed to educate the population to get vaccinated against COVID-19 (42). For example, some authors suggest that vaccination campaigns focusing on the older population should focus on making the risk of complications more salient (43). Basically, in moments like this pandemic, it is necessary for the authorities to incentive the "optimistic anxiety", described as a phenomenon in which "Citizens must be anxious enough to take the advice from the authorities to heart and optimistic enough as to feel that their actions make a difference - and therefore they will carry them out" (44).

Concerning political orientation, given its specific effect on the intention to vaccinate with SinovacCoronaVac, it must be taken into account by public agencies responsible for vaccination campaigns, since it can be a variable that negatively impacts the adherence of a portion of the population.

Vaccination campaigns need to be developed focusing on individuals of the right-wing political spectrum to overcome their resistance. Still, given the positive effect that celebrity endorsement has on forming a 
more favorable attitude towards vaccines (45), it would be crucial for campaigns to select celebrities who is favorable to the vaccine, but with a neutral or even right-wing political orientation.

This study has some limitations. The first one regarding the size and characteristics of the sample. Despite contemplating different educational levels and income, there is a huge concentration in the privileged classes, which do not necessarily represent the reality of the Brazilian elderly. Still, the fact that the research was carried out through the internet already causes a sample bias, as it leaves out participants who do not have access to this technology (46). However, this method was necessary, given the pandemic context, in which face-to-face participation could increase the respondents' risk of contamination. Also, due to the participant's age and the absence of face-to-face support to assist the respondent, the form had to be reduced, including the fewest questions possible. As a result, it was not possible to investigate other variables' role, which could also predict the intention to receive the vaccine, such as the tendency to nurture conspiracy beliefs and the level of credibility toward science. Therefore, these are variables that will need to be considered in future investigations.

In summary, this study contributes to public campaigns focusing on the mass immunization of the elderly against COVID-19 by emphasizing the importance of understanding the specificity of each vaccine separately. Also, it highlights the need to consider promotion aspects of a more favorable attitude towards vaccination, such as the positive effect of fear of COVID-19, and risk aspects for vaccination, such as the negative effect of vaccine uncertainty on the intention to vaccinate against this pandemic disease.

\section{Declarations}

\section{ACKNOWLEDGMENT:}

We want to thank the professionals of Clínica +60 Saúde (Belo Horizonte) and the Associação de Servidores Municipais de Betim (ASMUBE) for disseminating the survey form to the elderly. We also want to thank the National Council for Scientific and Technological Development - CNPq for the NMD Research Productivity scholarship.

\section{Conflict of interest:}

The authors declare that there is no conflict of interest.

\section{Author Contributions}

TVC conceived and searched the literature and organized and drafted the manuscript. FCMA, MRGD, TPBM, and NMD made substantial contributions and reviewed the manuscript critically for intellectual content. TVC, and NMD revised and approved the final manuscript for submission. All the authors contributed to the article and approved the submitted version. 


\section{References}

1. Dowd, J. B., Andriano, L., Brazel, D. M., Rotondi, V., Block, P., Ding, X., ... Mills, M. C. (2020).

Demographic science aids in understanding the spread and fatality rates of COVID-19. Proceedings of the National Academy of Sciences, 117(18), 9696-9698.

2. Liu, K., Chen, Y., Lin, R., \& Han, K. (2020). Clinical features of COVID-19 in elderly patients: A comparison with young and middle-aged patients. Journal of Infection, 80(6), e14-e18.

3. Araújo, M. P. D., Nunes, V. M. D. A., Costa, L. D. A., Souza, T. A. D., Torres, G. D. V., \& Nobre, T. T. X. (2021). Health conditions of potential risk for severe Covid-19 in institutionalized elderly people. PloS one, 16(1), e0245432.

4. Travassos, G. F., Coelho, A. B., \& Arends-Kuenning, M. P. (2020). The elderly in Brazil: demographic transition, profile, and socioeconomic condition. Revista brasileira de estudos de população, 37.

5. Daoust, J. F. (2020). Elderly people and responses to COVID-19 in 27 Countries. PloS one, 15(7), e0235590.

6. Dios-Guerra, C., Carmona-Torres, J. M., López-Soto, P. J., Morales-Cané, I., \& Rodríguez-Borrego, M. A. (2017). Prevalence and factors associated with influenza vaccination of persons over 65 years old in Spain (2009-2014). Vaccine, 35(51), 7095-7100.

7. Eilers, R., Krabbe, P. F., \& de Melker, H. E. (2015). Motives of Dutch persons aged 50 years and older to accept vaccination: a qualitative study. BMC Public Health, 15(1), 1-10.

8. Bacurau, A. G. D. M., \& Francisco, P. M. S. B. (2020). Reasons for non-vaccination against influenza among older adults with hypertension in Brazil: a cross-sectional study. Sao Paulo Medical Journal, 138(4), 322-325.

9. Wang, K., Wong, E. L. Y., Ho, K. F., Cheung, A. W. L., Yau, P. S. Y., Dong, D., ... Yeoh, E. K. (2021). Change of willingness to accept COVID-19 vaccine and reasons of vaccine hesitancy of working people at different waves of local epidemic in Hong Kong, China: Repeated cross-sectional surveys. Vaccines, 9(1), 62.

10. Mobbs, D. (2018). The ethological deconstruction of fear (s). Current opinion in behavioral sciences, 24, 32-37.

11. Tipples, J. (2018). Caution follows fear: Evidence from hierarchical drift diffusion modelling. Emotion, 18(2), 237.

12. Wake, S., Wormwood, J., \& Satpute, A. B. (2020). The influence of fear on risk taking: a metaanalysis. Cognition and Emotion, 34(6), 1143-1159.

13. Ahorsu, D. K., Lin, C. Y., Imani, V., Saffari, M., Griffiths, M. D., \& Pakpour, A. H. (2020). The fear of COVID-19 scale: development and initial validation. International journal of mental health and addiction, 1-9.

14. Shook, N. J., Sevi, B., Lee, J., Oosterhoff, B., \& Fitzgerald, H. N. (2020). Disease avoidance in the time of COVID-19: The behavioral immune system is associated with concern and preventative health behaviors. PloS one, 15(8), e0238015. 
15. Sultana, J., Mazzaglia, G., Luxi, N., Cancellieri, A., Capuano, A., Ferrajolo, C., ... Trifirò, G. (2020). Potential effects of vaccinations on the prevention of COVID-19: rationale, clinical evidence, risks, and public health considerations. Expert review of vaccines, 19(10), 919-936.

16. Harper, C. A., Satchell, L. P., Fido, D., \& Latzman, R. D. (2020). Functional fear predicts public health compliance in the COVID-19 pandemic. International journal of mental health and addiction, 1-14.

17. Flanagan, K. L., Best, E., Crawford, N. W., Giles, M., Koirala, A., Macartney, K., ... Wen, S. C. (2020). Progress and Pitfalls in the Quest for Effective SARS-CoV-2 (COVID-19) Vaccines. Frontiers in immunology, 11, 2410.

18. Kim, J. H., Marks, F., \& Clemens, J. D. (2021). Looking beyond COVID-19 vaccine phase 3 trials. Nature medicine, $1-7$.

19. Lancet, T. (2020). COVID-19 in Brazil:“So what?”. Lancet (London, England), 395(10235), 1461.

20. Green, J., Edgerton, J., Naftel, D., Shoub, K., \& Cranmer, S. J. (2020). Elusive consensus: Polarization in elite communication on the COVID-19 pandemic. Science Advances, 6(28), eabc2717.

21. Żuk, P., \& Żuk, P. (2020). Right-wing populism in Poland and anti-vaccine myths on YouTube: Political and cultural threats to public health. Global Public Health, 15(6), 790-804.

22. Claro, I. M., da Silva Sales, F. C., Ramundo, M. S., Candido, D. S., Silva, C. A.,de Jesus, J. G., ... Levi, J. E. (2021). Local Transmission of SARS-CoV-2 Lineage B.1.1. 7, Brazil, December 2020. Emerging infectious diseases, 27(3), 970.

23. Ahorsu, D. K., Lin, C. Y., Imani, V., Saffari, M., Griffiths, M. D., \& Pakpour, A. H. (2020). The fear of COVID-19 scale: development and initial validation. International journal of mental health and addiction, 1-9.

24. Giordani, R. C. F., Zanoni da Silva, M., Muhl, C., \& Giolo, S. R. (2020). Fear of COVID-19 scale: Assessing fear of the coronavirus pandemic in Brazil. Journal of Health Psychology, 1359105320982035.

25. Cavalheiro, F. R. S., \& Sticca, M. G. (2020). Adaptation and Validation of the Brazilian Version of the Fear of COVID-19 Scale. International Journal of Mental Health and Addiction, 1-9.

26. Inbar, Y., Pizarro, D., lyer, R., \& Haidt, J. (2012). Disgust sensitivity, political conservatism, and voting. Social Psychological and Personality Science, 3(5), 537-544.

27. Korang, S. K., Juul, S., Nielsen, E. E., Feinberg, J., Siddiqui, F., Ong, G., ... Thomsen,A. R. (2020). Vaccines to prevent COVID-19: a protocol for a living systematic review with network meta-analysis including individual patient data (The LIVING VACCINE Project). Systematic reviews, 9(1), 1-15.

28. Wang, K., Wong, E. L. Y., Ho, K. F., Cheung, A. W. L., Yau, P. S. Y., Dong, D., ...Yeoh, E. K. (2021). Change of willingness to accept COVID-19 vaccine and reasons of vaccine hesitancy of working people at different waves of local epidemic in Hong Kong,China: Repeated cross-sectional surveys. Vaccines, 9(1), 62.

29. Bacurau, A. G. D. M., \& Francisco, P. M. S. B. (2020). Reasons for non-vaccination against influenza among older adults with hypertension in Brazil: a cross-sectional study. Sao Paulo Medical Journal, 138(4), 322-325. 
30. Shook, N. J., Sevi, B., Lee, J., Oosterhoff, B., \& Fitzgerald, H. N. (2020). Disease avoidance in the time of COVID-19: The behavioral immune system is associated with concern and preventative health behaviors. PloS one, 15(8), e0238015.

31. Zareipour, M. A., Ardakani, M. F., Moradali, M. R., Jadgal, M. S., \& Movahed, E. (2020). Determinants of COVID-19 Prevention Behavior in the Elderly in Urmia: Application of Health Belief Model. Open Access Macedonian Journal of Medical Sciences, 8(T1), 646-650.

32. Mobbs, D. (2018). The ethological deconstruction of fear (s). Current opinion in behavioral sciences, $24,32-37$.

33. Tipples, J. (2018). Caution follows fear: Evidence from hierarchical drift diffusion modelling. Emotion, 18(2), 237.

34. Wake, S., Wormwood, J., \& Satpute, A. B. (2020). The influence of fear on risk taking: a metaanalysis. Cognition and Emotion, 34(6), 1143-1159.

35. Barberia, L. G., \& Gómez, E. J. (2020). Political and institutional perils of Brazil's COVID-19 crisis. The Lancet, 396(10248), 367-368.

36. Voysey, M., Clemens, S. A. C., Madhi, S. A., Weckx, L. Y., Folegatti, P. M., Aley,P. K., ... Bijker, E. (2021). Safety and efficacy of the ChAdOx1 nCoV-19 vaccine (AZD1222)against SARS-CoV-2: an interim analysis of four randomised controlled trials in Brazil,South Africa, and the UK. The Lancet, 397(10269), 99-111.

37. Zhang, Y., Zeng, G., Pan, H., Li, C., Hu, Y., Chu, K., ... Zhu, F. (2021). Safety, tolerability,and immunogenicity of an inactivated SARS-CoV-2 vaccine in healthy adults aged $18-59$ years: a randomised, double-blind, placebo-controlled, phase 1/2 clinical trial. The Lancet Infectious Diseases, 21(2), 181-192.

38. Soiza, R. L., Scicluna, C., \& Thomson, E. C. (2021). Efficacy and safety of COVID-19 vaccines in older people. Age and Ageing, 50(2), 279-283.

39. Petersen, M. B., Bor, A., Jørgensen, F. J., \& Lindholt, M. F. (2020). Transparency Is Necessary but Not Sufficient to Reduce Skepticism About a COVID-19 Vaccine. PsyArxiv.

40. Seethaler, S. L. (2016). Shades of grey in vaccination decision making: Tradeoffs, heuristics, and implications. Science Communication, 38(2), 261-271.

41. Weisberg, D. S., Landrum, A. R., Hamilton, J., \& Weisberg, M. (2020). Knowledge about the nature of science increases public acceptance of science regardless of identity factors. Public Understanding of Science, 0963662520977700

42. DeRoo, S. S., Pudalov, N. J., \& Fu, L. Y. (2020). Planning for a COVID-19 vaccination program. Jama, 323(24), 2458-2459.

43. Eilers, R., Krabbe, P. F., \& de Melker, H. E. (2015). Motives of Dutch persons aged 50 years and older to accept vaccination: a qualitative study. BMC Public Health, 15(1), 1-10.

44. Petersen, M. B. (2020). The unpleasant truth is the best protection against coronavirus. Politiken, (March 9). 
45. Alatas, V., Chandrasekhar, A. G., Mobius, M., Olken, B. A., \& Paladines, C. (2019). When celebrities speak: A nationwide twitter experiment promoting vaccination in Indonesia (No. w25589). National Bureau of Economic Research.

46. Lourenco, S. F., \& Tasimi, A. (2020). No participant left behind: conducting science during COVID-19. Trends in Cognitive Sciences, 24(8), 583-584.

\section{Tables}

Table 1. Predictor variables to the intention to be vaccinated with the Oxford-AstraZeneca vaccine

\begin{tabular}{|c|c|c|c|c|c|}
\hline & \multirow[b]{2}{*}{ Predictors } & Standardized coefficients & \multirow[t]{2}{*}{$t$} & \multirow[t]{2}{*}{$p$} & \multirow[t]{2}{*}{$R^{2} a d j$} \\
\hline & & Beta & & & \\
\hline \multirow[t]{2}{*}{ Model 01} & (Constant) & & 45.783 & 0.000 & 0.255 \\
\hline & Uncertainty Index & -0.507 & -10.622 & 0.000 & \\
\hline \multirow[t]{3}{*}{ Model 02} & (Constant) & & 18.236 & 0.000 & 0.266 \\
\hline & Uncertainty Index & -0.479 & -9.837 & 0.000 & \\
\hline & Fear of COVID-19 & 0.118 & 2.421 & 0.016 & \\
\hline
\end{tabular}

Table 2. Predictor variables to the intention to be vaccinated with the Sinovac-CoronaVac

\begin{tabular}{|c|c|c|c|c|c|}
\hline & \multirow[b]{2}{*}{ Predictors } & Standardized coefficients & \multirow[t]{2}{*}{$t$} & \multirow[t]{2}{*}{$p$} & \multirow[t]{2}{*}{$R^{2} a d j$} \\
\hline & & Beta & & & \\
\hline \multirow[t]{2}{*}{ Model 01} & (Constant) & & 45.783 & 0.000 & 0.431 \\
\hline & Uncertainty Index & -0.658 & -15.780 & 0.000 & \\
\hline \multirow[t]{3}{*}{ Model 02} & (Constant) & & 29.082 & 0.000 & 0.443 \\
\hline & Uncertainty Index & -0.603 & -13.167 & 0.000 & \\
\hline & Political Orientation & -0.128 & -2.791 & 0.006 & \\
\hline \multirow[t]{4}{*}{ Model 03} & (Constant) & & 15.496 & 0.000 & 0.493 \\
\hline & Uncertainty Index & -0.586 & -12.683 & 0.000 & \\
\hline & Political Orientation & $-0,118$ & -2.575 & 0.010 & \\
\hline & Fear of COVID-19 & 0.089 & 2.097 & 0.037 & \\
\hline
\end{tabular}

\section{Figures}




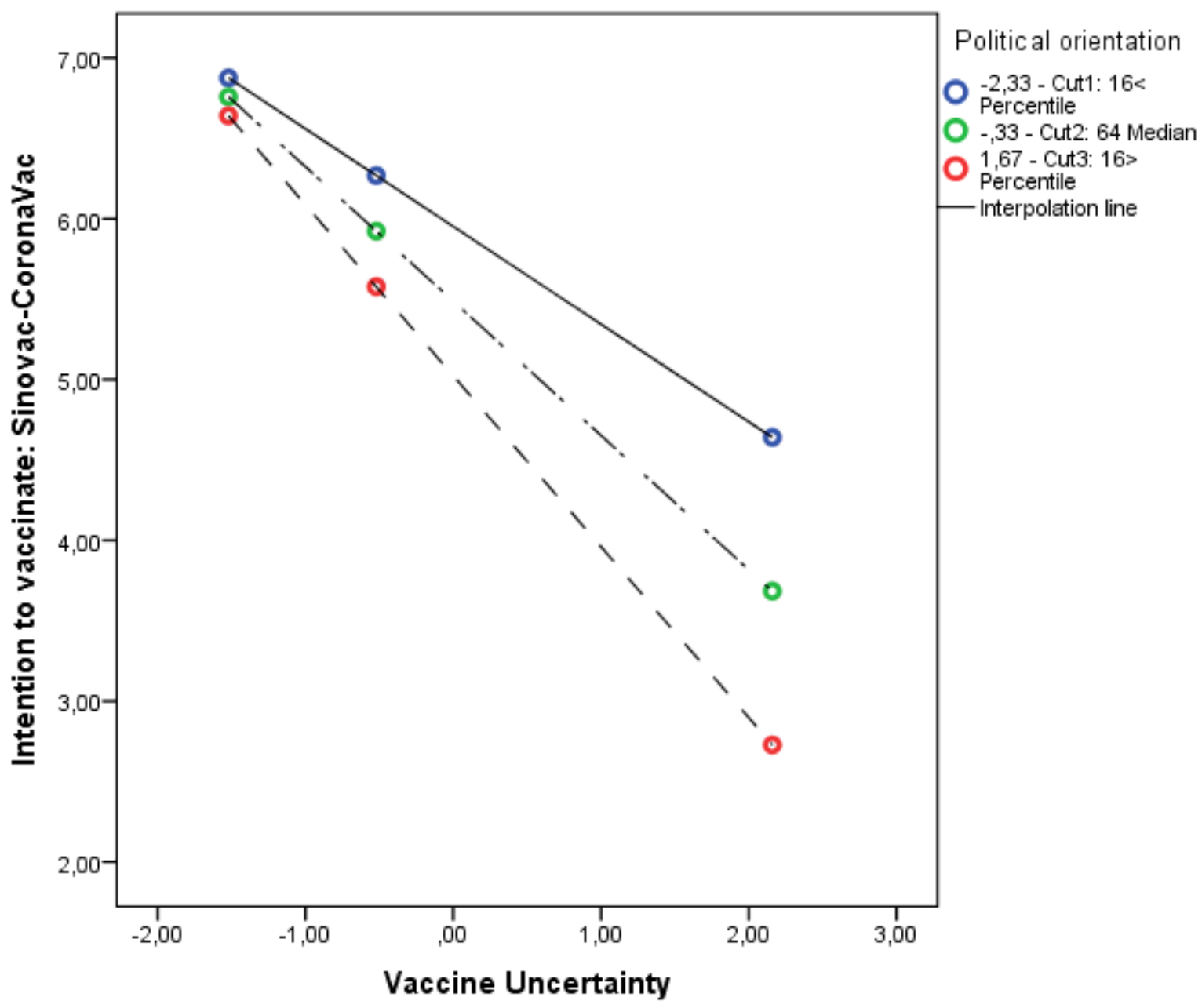

Figure 1

Moderation of Political orientation on the relationship between Vaccine Uncertainty Index and Vaccination intention (Sinovac-CoronaVac). 